\title{
A Research on VANET: Various Broadcasting and Clustering Techniques
}

\author{
D. Kalaivani, Rajkumar S
}

\begin{abstract}
In present years, there is a rapid increase in number of vehicles flying on the road. The focus is to improve the road safety and navigations standards with the help of an intelligent transport system. There is a need for novel applications and services in the vehicular environment for security and comfort. Technical advancement has been developed to predict road accidents, to prevent collisions, to understanding road conditions, to access uninterrupted internet facilities to expand the transmission range, to extend the storage capacity and to avoid the interference of wireless links. The idea of this paper work is to develop an intelligent transport model to enhance the road safety and navigation process. There are many approaches in the $n$ communication. Effective and efficient techniques are developed to detect the road conditions with the help of vehicular communication. This paper gives the background of intelligent vehicle transport system. Various literature on VANET modelling, clustering and its based algorithms are discussed. Followed by a review of hybrid cluster algorithms, MAC protocols for VANET road safety applications, multi-hop and multi-level broadcast protocol are discussed.
\end{abstract}

Keywords:-VANET, Intelligent transportation system (ITS), clustering, broadcast protocols, MAC Protocols.

\section{INTRODUCTION}

In the $20^{\text {th }}$ century, due to the growth in number of vehicles, transport infrastructure was, built which was appropriate for handling road traffic congestion, road accidents, road transportation delays etc. During that era, significance was, given to constructing new roads rather than ITS technological developments. Electric traffic signal was the very first ITS technical development in 1928. Later on, an Automated Highway Systems (AHS) was, introduced for highway traffic. In the 1960's traffic signals, where first controlled by the computers. Figueiredoet. al (2001) introduced a system to guide an electronic route for two-way road vehicle communication to give route assistance. In 1970s, based on the real traffic conditions a dynamic route assistance system,Koshi (1989), French R.L (1999) developed a control systemand information system for automobile traffic and autofahrerleitwas introduced

\section{RELATED WORK\& RESULTS}

A detailed study regarding the universal characteristics of few major ITS categories are discussed.

Shibata, J., \& French, R. (1999) introduced the first category is the Advanced Traffic Management Systems (ATMS) were introduced to enhance the service quality of

Revised Manuscript Received on April 12, 2019.

D. Kalaivani,Research Scholar, School of Computer Science and Engineering, Vellore Institute of Technology, Vellore Tamil Nadu, India. (dkalaivanisai@gmail.com)

Dr. Rajkumar S, Associate Professor School of Computer Science and Engineering, Vellore Institute of Technology, Vellore Tamil Nadu, India. (rajkumars@vit.ac.in) road traffic and to minimize the road traffic delays. Next, Advanced Travellers Information System (ATIS) was, introduced to offer real-time traffic conditions. Based on the current traffic situations the travellers can avoid traffic congestions by taking an alternate road to reach the destination. Commercial Vehicles Operations (CVO) system uses ITS technology to improve the road safety for the commercial vehicles. To improve the operations in mass transport vehicles such as buses and trains an Advanced Public Transportations Systems (APTS) uses electronics technology. Advanced Vehicle Control Systems (AVCS) uses sensors to alert drivers about the environment Advanced Rural Transports Systems (ARTS) was designed especially for the rural areas.

Many literatures about the ITS, VANET and Clustering are discussed in this review paper. This study helps to influence the centre ideas and to have deep understanding of the technologies. The review of literature helps to narrow down the research that helps in developing the proposed system. The following sections describe a detailed review of the existing techniques in VANET clustering, and various broadcast protocols in VANETs.

\subsection{Related work on VANETs Clustering}

Gupta $N$ et. al (2015) proposed a cognitive algorithm for efficient utilization of bandwidth and consistent data delivery. The algorithm that was designed in the vehicular system allowed a dynamic allocation of channels in available bands. Cluster Cognitive Medium Access Control (CCMAC) protocol allocates channels to ensure proactively switching of the messages dynamically and guarantee distribution of safety messages. CCMAC protocol implements mobility based clustering algorithm to arrange the messages in order of node speed. Service channels are proposed in the real-time message delivery to lengthen the delivery of safety messages within the band and for the delivery of non-safety messages the bands are changed. For $\mathrm{V} 2 \mathrm{~V}$ communications, the proposed protocol is, evaluated under changing circumstances for safety and non-safety applications.

Kwon J.H et.al (2016) developed a neighbour stabilitybased VANET clustering (NSVC) to reduce the topology changes and to minimize the delay in cluster formation. The NSVC is proposed for the urban vehicles due to its high mobility and unpredicted route during movement. The vehicle-to-vehicle communication and vehicle-toinfrastructure communication should consider repeated changes in the topology of VANETs. Transmission 
scheduling method supports emergency message transmissions. Based on the neighbour stability, it makes decisions whether or not to execute the cluster head election procedures. For high priority data transmission, polling based channel access is provided. The network performance is better in NSVC compared to existing methods.

Wang $\mathrm{H}$ et. al (2015) proposed a comprehensive analysis model which incorporates three vital factors into a single model in VANETs. An idle state is, introduced in an unsaturated VANET cluster with a Markov chain. The Vehicle mobility and wireless channel fading are, combined by joint distribution method for the inter-vehicle distance. In general, VANETs perform grouping of vehicles to assist communication. The cluster design such as size, geographical distance, vehicle speed, traffic volume and window size has major impacts on communication quality. Network performance is measured, in terms of packet loss probability and system throughput.

SahooA et. al (2016) attempted to optimize a routing algorithm for VANETs. Nature inspired optimization technique such as Ant Colony Optimization (ACO) is applied for clustering technique. To develop ITS, and to improve the safety standards a unique type of MANET is used as a routing protocol. ACO and zone based clustering algorithm are combined to enhance the results.

Da Silva et. al (2016) proposed a distributed strategy for vehicular networks based on Trajectory aware Content (TraC). This strategy combines Content-Centric Networks (CCNs) and users' trajectory aware content. To increase the content delivery probability in vehicular environment, it uses proactive caching in the network formed by Access Points (APs). Two forwarding strategies are, proposed for vehicles between access points and neighbourhood discovery protocol for CCNs. Three vehicle scenarios are evaluated, such as highway traffic, urban traffic and rushhour situation from a dataset. Evaluations are, based on the content delivery ratio, growing number of vehicles, network load and TraC performance and $\mathrm{CCN}$ versions for wireless environments.

UcarS et. al (2016) proposed a hybrid architecture named Vehicular Multihop algorithm for Stable Clustering and Long Term Evolution (VMaSC-LTE). This above proposal aim to achieve low delay and Data Packet Delivery Ratio (DPDR). The goal is to minimize the cluster head and maximize the cluster stability in the cellular architecture. The performance analysis of multi-hop cluster based IEEE 802.11p-LTE includes DPDR, cluster stability, delay and control overhead.

Shanmugasundaram G et. al (2017) developed a VANET multilevel cluster algorithm which is novel in grouping vehicles which shows related mobility pattern in single cluster. To form an effective cluster, first cluster process is initiated, then cluster head selected, to minimize the cluster overhead slave selection technique is introduced, the final step is merge the cluster. The results illustrates that the proposed technique maximises the cluster spantime and minimises the transmission time of the messages between nodes. The communication between the cluster heads is achieved along with an increase in cluster stability.

Bi Y et. al (2016) proposed a protocol named Urban Multi-hop Broadcast Protocol (UMBP) to distribute crisis

messages. UMBP consists of, a forwarding node selection system, to reduce the transmission delay of urgent situation messages and to minimize redundant messages. The novel system develops an iterative partition, mini-slot and blackburst to, rapidly select remote neighbouring nodes. Three schemes for broadcast such as directional, bidirectional and multi-directional broadcast are designed based on the novel system to distribute emergency messages. The protocol in terms of one-hop delay and message transmission speed was analysed, and a model was developed to measure the performance.

Omar et. al (2016) discussed the feasibility of safety applications in cellular network technology and in wireless communication standards: IEEE 802.11 p known as Wireless Access in Vehicular Environments (WAVE). A protocol named VeMAC, is developed for VANET safety applications which uses Time-Division Multiple Access (TDMA) protocol. VeMAC, is proposed to overcome the limitations of the existing work in VANETs by broadcasting reliable safety messages. The performances of IEEE 802.11p standards and VeMAC protocol are compared through a computer simulator. The performance of the protocol is compared in terms of event driven safety messages in different highway and urban situations. Recent developments in VeMAC protocol include on-road demonstrations of safety applications.

Bian C et. al (2015) proposed a technique to enhance the Named Data Networking (NDN) architecture to maintain a productive content delivery in urban VANETs. NDN is an architecture of data-centric to achieve the content retrieval and distribution through named data delivery. A geo-based NDN design approach is proposed, to overcome the challenges caused by high movement of vehicles in urban VANETs. When NDN is, applied to VANETs, cache redundancy occurs so heuristic approaches are, proposed to remove the cache redundancy problem. Simulation results shows that the enhanced NDN's approach data distribution is better when compared to the original NDN approach.

OcheM et. al (2015) conducted an experiment to verify the feasibility of Internet Protocol Television (IPTV) services above VANETs. Analytical model is, derived to measure the quality of IPTV services. Challenges such as topology changes in VANETs, real time streaming of IPTV traffic with high bandwidth and service quality are assessed. Various performance metrics are video quality, moving image quality, peak signal to noise ratio. The relationship between the IPTV traffic and the variables of each parameter is assessed. Quality of service parameters such as bandwidth allocation, delay and loss control is evaluated for better quality of IPTV traffic.

RautS et. al (2015) proposed a VANET based vehicle collision prediction system to avoid collision. To predict the probability at highway intersection Intelligent Control Unit (ICU) and $\mathrm{V} 2 \mathrm{~V}$ communication is used. The proposed evaluation system monitors and transmits beacon messages to the possible colliding vehicles. Based on calculated metric alert messages are sent to the vehicles which are in danger. 


\subsection{Related work on broadcast approaches in VANETs}

Wu et. al (2013) developed a Backbone Based Broadcast (BBBR) protocol for VANETs. The proposed system dynamically selects the backbone vehicles to distribute information. The proposed approach reduces contention time in the MAC layer at every node by maintaining high packet distribution ratio.

Dawood HS \& Wang Y (2013) proposed an Efficient Emergency Message Broadcasting (EEMB) system in VANETs. The vehicles located in the risk zone are broadcasted with emergency messages. The broadcasted emergency message avoids multiple accidents and reduces traffic jam. Emergency messages are broadcasted at rapid speed with less number of overheads. The best relay(s) will selected by the accident vehicle and the emergency message will be broadcasted. The relay will select the next best relay and rebroadcast the emergency message. This procedure continues until the urgent or alert message covers the danger zone vehicles. The proposed system reduces the overheads of signals by prediction method.

Choi J et. al (2014) proposed a broadcast system for VANETs named virtual slotted p-persistence scheme. A neighbour table maintains information about the vehicle's position in the neighbourhood. A virtual slot is created as soon as a vehicle receives emergency messages. The proposed approach, assure that even the farthest group receives the broadcasted messages.
Huang J et. al (2014) proposed a IEEE802.11 based multi-hop broadcast protocol to deal with the emergency message distribution. A forwarder is selected in proposed method based on the vehicle density. Simulation results shows that the proposed technique reduces the transmission collision and lowers the broadcast delay.

Hoque $\mathrm{F}$ et. al (2014) proposed a two way intelligent broadcasting approach to send effective warning messages for avoiding collision. The emergency detecting vehicle sends emergency warning messages. In multi-hop mechanism, the warning messages are, broadcasted due to limited transmission range. The inter-vehicle delay and endto-end delay, is minimized in the proposed two-way approach when compared to the existing algorithm. Hence avoids the chain collision.

Fan X (2015) proposed a multi-hop broadcast scheme for road side unit and vehicle to infrastructure communications. The emergency messages are delivered at a high delivery rate and delay is minimal. The network utilization is better in the proposed system regardless of the number of vehicles. Pesavento D et.al (2013) designed a naming system to insert a location in the Named Data Networking model for effective mapping of bi-dimensional geographic areas into uni-dimensional naming system. A naming design system can guide to obtain high cache hit ratio, efficient bandwidth utilization and least network latency. It's designed specifically for interaction between hosts and for getting the content specific to geographic region.

Table 2.1 Review of Literature about Clustering Techniques

\begin{tabular}{|c|c|c|c|}
\hline Author & Clustering Technique & Methodology Used & Limitations \\
\hline Gupta $\mathrm{N}$ et. al (2015) & $\begin{array}{l}\text { Mobility based clustering } \\
\text { algorithm to prioritize } \\
\text { messages in the order of node } \\
\text { speed }\end{array}$ & $\begin{array}{l}\text { Cluster Cognitive Medium } \\
\text { Access Control (CCMAC) } \\
\text { protocol }\end{array}$ & $\begin{array}{l}\text { Lengthen the delivery of safety } \\
\text { messages within the band }\end{array}$ \\
\hline Kwon J.H et.al (2016) & $\begin{array}{l}\text { Neighbor stability-based } \\
\text { VANET clustering (NSVC) }\end{array}$ & $\begin{array}{l}\text { Transmission scheduling } \\
\text { method supports emergency } \\
\text { message }\end{array}$ & $\begin{array}{l}\text { To reduce the topology } \\
\text { changes and to minimize the } \\
\text { delay in cluster formation. }\end{array}$ \\
\hline Wang $\mathrm{H}$ et. al (2015) & $\begin{array}{l}\text { VANETs group vehicles into } \\
\text { clusters to assist } \\
\text { communication. }\end{array}$ & $\begin{array}{l}\text { Comprehensive analysis } \\
\text { model }\end{array}$ & $\begin{array}{l}\text { Packet loss probability and } \\
\text { system throughput. }\end{array}$ \\
\hline SahooA et. al (2016) & $\begin{array}{l}\text { To optimize a routing zone } \\
\text { clustering algorithm for } \\
\text { VANETs using ACO. }\end{array}$ & Ant Colony Optimization & Zone based \\
\hline Da Silva et. al (2016) & $\begin{array}{l}\text { Uses proactive caching in the } \\
\text { network formed by Access } \\
\text { Points (APs). }\end{array}$ & $\begin{array}{l}\text { Trajectory aware Content } \\
\text { (TraC). }\end{array}$ & $\begin{array}{l}\text { highway traffic, urban traffic } \\
\text { and rush-hour situation from a } \\
\text { dataset. }\end{array}$ \\
\hline UcarS et. al (2016) & $\begin{array}{l}\text { Vehicular Multihop algorithm } \\
\text { for Stable Clustering and } \\
\text { Long Term Evolution }\end{array}$ & $\begin{array}{l}\text { multi-hop cluster based IEEE } \\
802.11 \text { p-LTE }\end{array}$ & $\begin{array}{l}\text { Cluster stability, delay and } \\
\text { control overhead. }\end{array}$ \\
\hline $\begin{array}{l}\text { Shanmugasundaram G } \\
\text { et. al (2017) }\end{array}$ & $\begin{array}{l}\text { VANET multilevel cluster } \\
\text { algorithm }\end{array}$ & $\begin{array}{l}\text { cluster overhead slave } \\
\text { selection technique is } \\
\text { introduced, }\end{array}$ & $\begin{array}{l}\text { cluster head-to-head } \\
\text { communication }\end{array}$ \\
\hline Bi Y et. al (2016) & $\begin{array}{l}\text { forwarding node selection } \\
\text { system }\end{array}$ & UMBP & $\begin{array}{l}\text { one-hop delay and message } \\
\text { transmission speed }\end{array}$ \\
\hline
\end{tabular}




\begin{tabular}{|l|l|l|l|}
\hline Omar et. al (2016) & $\begin{array}{l}\text { Wireless Access in Vehicular } \\
\text { Environments (WAVE). }\end{array}$ & VeMAC protocol & on-road demonstrations \\
\hline Bian C et. al (2015) & $\begin{array}{l}\text { Named Data Networking } \\
\text { (NDN) architecture }\end{array}$ & $\begin{array}{l}\text { geo-based NDN design } \\
\text { approach }\end{array}$ & $\begin{array}{l}\text { Urban VANET, to remove the } \\
\text { cache redundancy }\end{array}$ \\
\hline OcheM et. al (2015) & $\begin{array}{l}\text { Internet Protocol Television } \\
\text { services }\end{array}$ & Analytical model & $\begin{array}{l}\text { bandwidth allocation, delay } \\
\text { and loss control }\end{array}$ \\
\hline RautS et. al (2015) & collision prediction system & $\begin{array}{l}\text { probability at highway } \\
\text { intersection Intelligent } \\
\text { Control Unit (ICU) }\end{array}$ & $\begin{array}{l}\text { monitors and transmits beacon } \\
\text { messages }\end{array}$ \\
\hline
\end{tabular}

Table 2.2 Review of Literature about Broadcasting Technique

\begin{tabular}{|c|c|c|c|}
\hline Author & Broadcasting Technique & Methodology Used & Limitations \\
\hline Wu et. al (2013) & BBBR protocol & $\begin{array}{l}\text { Dynamically selects the backbone } \\
\text { vehicles to distribute information. }\end{array}$ & MAC layer contention \\
\hline $\begin{array}{l}\text { Dawood HS \& Wang Y } \\
\text { (2013) }\end{array}$ & EEMB system & $\begin{array}{l}\text { Risk zone are broadcasted with } \\
\text { emergency messages. Prediction method }\end{array}$ & $\begin{array}{l}\text { relay, rebroadcast, } \\
\text { overheads of signals }\end{array}$ \\
\hline Choi J et. al (2014) & $\begin{array}{l}\text { Virtual slotted p- } \\
\text { persistence scheme. }\end{array}$ & $\begin{array}{l}\text { Neighbor table maintains information } \\
\text { about the vehicle's position in the } \\
\text { neighborhood. }\end{array}$ & farthest group \\
\hline Huang J et. al (2014) & $\begin{array}{l}\text { IEEE802.11 based multi- } \\
\text { hop broadcast protocol }\end{array}$ & $\begin{array}{l}\text { Forwarder is selected based on the } \\
\text { vehicle density }\end{array}$ & $\begin{array}{l}\text { Lowers the broadcast } \\
\text { delay. }\end{array}$ \\
\hline Hoque $\mathrm{F}$ et. al (2014) & $\begin{array}{l}\text { two way intelligent } \\
\text { broadcasting approach }\end{array}$ & multi-hop mechanism & chain collision \\
\hline Fan X (2015) & $\begin{array}{l}\text { multi-hop broadcast } \\
\text { scheme }\end{array}$ & vehicle to infrastructure communications & network utilization \\
\hline $\begin{array}{l}\text { Pesavento D et.al } \\
(2013)\end{array}$ & $\begin{array}{l}\text { Named Data Networking } \\
\text { model }\end{array}$ & $\begin{array}{l}\text { Bi-dimensional geographic areas into } \\
\text { uni-dimensional naming system. }\end{array}$ & $\begin{array}{l}\text { Content specific to } \\
\text { geographic region. }\end{array}$ \\
\hline
\end{tabular}

\section{SUMMARY}

This paper gives the background of intelligent vehicle transport system. Various literatures on VANET modelling and clustering are discussed. A detailed review of clustering technique in VANETs, its broadcasting approach of various application models and VANET cluster based algorithms are discussed. The technological approaches to solve the transmission delay problem in VANETs are discussed. Literatures on MAC protocols for VANET road safety applications, multi-hop and multi-level broadcast protocol, named data centric network, content centric network are also discussed.

\section{REFERENCES}

1. Figueiredo, Lino, Isabel Jesus, JA Tenreiro Machado, Jose Rui Ferreira, and JL Martins De Carvalho. "Towards the development of intelligent transportation systems." In ITSC 2001. 2001 IEEE Intelligent Transportation Systems. Proceedings (Cat. No. O1TH8585), pp. 1206-1211. IEEE, 2001.

2. Koshi, M. "Development of the advanced vehicle road information systems in japan-the cacs project and after." JSK International Symposium-Technological Innovations for Tomorrow's Automobile Traffic and Driving Information Systems, Japan. 1989.

3. French, Robert L. "The IEEE and ITS." IEEE Intelligent Systems 6 (1999): 75-77.

4. Shibata, J., and R. French. "Progress in intelligent transportation systems in the US, Europe and Japan." ISATA magazine. R\&D French Associates, 1999. 27-29.

5. Gupta, Nishu, ArunPrakash, and Rajeev Tripathi. "Medium access control protocols for safety applications in
Vehicular Ad-Hoc Network: A classification and comprehensive survey." Vehicular Communications 2.4 (2015): 223-237.

6. Kwon, Jung-Hyok, et al. "Neighbor stability-based VANET clustering for urban vehicular environments." The Journal of Supercomputing 72.1 (2016): 161-176.

7. Wang, Huixian, et al. "VANET modeling and clustering design under practical traffic, channel and mobility conditions." IEEE Transactions on Communications 63.3 (2015): 870-881.

8. Sahoo, Arundhati, et al. "An optimized cluster based routing technique in VANET for next generation network." Information Systems Design and Intelligent Applications. Springer, New Delhi, 2016. 667-675.

9. da Silva, Vitor Borges Coutinho, Miguel Elias M. Campista, and Luís Henrique MK Costa. "TraC: A Trajectory-aware Content distribution strategy for vehicular networks." Vehicular Communications 5 (2016): 18-34.

10. Ucar, Seyhan, SinemColeriErgen, and OznurOzkasap. "Security vulnerabilities of IEEE 802.11 p and visible light communication based platoon." 2016 IEEE Vehicular Networking Conference (VNC). IEEE, 2016.

11. Shanmugasundaram, G., et al. "A Multilevel Clustering Using Multi-hop and Multihead in VANET." Proceedings of the International Conference on Data Engineering and Communication Technology. Springer, Singapore, 2017.

12. Bi, Yuanguo, et al. "A multi-hop broadcast protocol for emergency message dissemination in urban vehicular ad hoc networks." IEEE Transactions on Intelligent Transportation Systems 17.3 (2015): 736-750. 
13. Omar, Hassan Aboubakr, Ning Lu, and WeihuaZhuang. "Wireless access technologies for vehicular network safety applications." IEEE Network 30.4 (2016): 22-26.

14. Bian, Chaoyi, et al. "Boosting named data networking for data dissemination in urban VANET scenarios." Vehicular Communications 2.4 (2015): 195-207.

15. Oche, Michael, RafidahMd Noor, and Johnson IhyehAghinya. "Network centric QoS performance evaluation of IPTV transmission quality over VANETs." Computer Communications 61 (2015): 34-47.

16. Raut, Swati B., Preeti R. Bajaj, and Latesh G. Malik. "Prediction of vehicle collision probablity at intersection using V2V communication." Int. J. Sci. Eng. Res 6 (2015): 295-300.

17. Wu, Celimuge, Satoshi Ohzahata, and Toshihiko Kato. "Data dissemination with dynamic backbone selection in vehicular ad hoc networks." 2013 IEEE 78th Vehicular Technology Conference (VTC Fall). IEEE, 2013.

18. Dawood, Hayder Salman, and Yuming Wang. "An efficient emergency message broadcasting scheme in vehicular ad hoc networks." International journal of distributed sensor networks9.11 (2013): 232916.

19. Choi, Jae-In, Jae-Choong Nam, and You-Ze Cho. "Robust broadcast scheme regardless of vehicle distribution in vehicular ad hoc networks." EURASIP journal on wireless communications and networking 2014.1 (2014): 133.

20. Huang, Jiawei, Yi Huang, and Jianxin Wang. "Vehicle density based forwarding protocol for safety message broadcast in VANET." The scientific world journal 2014 (2014).

21. Hoque, Faika, and Sungoh Kwon. "An emergency packet forwarding scheme for $\mathrm{V} 2 \mathrm{~V}$ communication networks." The scientific world journal 2014 (2014).

22. Fan, Xing, et al. "Road side unit assisted stochastic multihop broadcast scheme for instant emergency message propagation." 2015 17th International Conference on Advanced Communication Technology (ICACT). IEEE, 2015.

23. Pesavento, Davide, et al. "A naming scheme to represent geographic areas in NDN." 2013 IFIP Wireless Days (WD). IEEE, 2013.

24. Omar, Hassan Aboubakr, WeihuaZhuang, and $\mathrm{Li} \mathrm{Li}$. "VeMAC: A TDMA-based MAC protocol for reliable broadcast in VANETs." IEEE transactions on mobile computing 12.9 (2012): 1724-1736. 